\title{
Development of a red-light running violation index model for signalized intersections
}

\author{
S. A. Arhin ${ }^{1}$, E. C. Noel $^{1}$, L. Williams ${ }^{1} \&$ M. Lakew ${ }^{2}$ \\ ${ }^{1}$ Howard University, USA \\ ${ }^{2}$ District Department of Transportation, USA
}

\begin{abstract}
The installation of Red-Light Cameras (RLCs) is often based on frequencies of Red Light Violations at signalized intersections. Since frequencies could be influenced by geometrical features of intersecting roadways and overall vehicular volumes (or exposure) there is doubt about their appropriateness in making decisions regarding the installation of RLCs. Inappropriate installation could result in unintended consequences such as increasing the frequency of some types of crashes. In order to eliminate the potential for bias with the use of frequencies as a means of deciding which intersections may need RLCs, a RedLight Violation Index (RLVI) is introduced for dense urban environments. There is no red-light violation or red light crash threshold in the District of Columbia above which red light cameras should be considered for installation. In this research, a model for background or base RLVI was established which could assist engineers in determining the expected potential for red light running at intersections based on engineering properties, without the use of red-light running frequencies or crash records. A RLVI probabilistic regression model was developed based on five intersection independent variables: vehicles per hour green, lane configuration, clearance distance, duration of green and posted speed limit. The results showed a statistically significant regression model with an $R^{2}$ of $81 \%$, at a $5 \%$ level of significance.
\end{abstract}

Keywords: red-light running, intersection safety.

\section{Introduction}

Between 1999 and 2010, the Metropolitan Police Department installed approximately 50 Red-Light Cameras (RLCs) in the District of Columbia (DC). 
The installations were in response to a perceived high rate of red-light violations at intersections, and a need mitigate the red-light violation (RLV) problem. There is no established basis in the District of Columbia for determining when the RLV experience of an intersection is beyond expectation. A set of criteria is essential to enable the City to objectively prioritize the implementation of strategies for reducing RLV, including engineering and public awareness programs. Several measures are documented in the general literature on red-light running issues. They include the number of violations per certain volume of vehicles, the number of violations per unit time, the number of violations per cycle, as well as complex algorithms for calculating a RLV rate.

A fundamental concern about using the volume-based RLV rate for categorizing the status of RLV of intersections is that motorists who never had a chance to violate (those who crossed on green or amber, and those who never got to the stop line) are included in the factors used in characterizing the RLV status of intersection approaches. In addition, some agencies use the number of RLV citations or RLV crashes in characterizing RLV status, although most RLVs are not recorded and are excluded from crash reports. Further, RLV per unit time period ignores traffic exposure. There is, therefore, the need for a method for establishing the RLV status of intersections. Thus a Red-Light Violation Index (RLVI) is established in this research. The method for obtaining the RLVI of an intersection should be easy to understand, and measureable from field observations. Most agencies do not routinely monitor trends in RLV at critical intersections to determine whether implemented treatments are successful. The RLVI developed serves as a basis for estimating the minimal level of RLV for typical intersections in the City. Knowledge of the background RLV status has two purposes:

1. It enables the District Department of Transportation (DDOT) to establish change via monitoring intersections and comparing values, and

2. It provides a basis for comparing the status with a RLV threshold, i.e. the RLVI.

The extent to which observed RLV status exceeds the standard could be used as a basis for installing RLR cameras at intersections. This research explores the relationship between signalized intersection-related variables and RLV, and develops a model that could estimate the background violation potential for intersections within DC. A major portion of the effort was geared towards determining whether or not a statistically significant, probabilistic model could be developed to determine the background violation potential as a function of standard intersection-related variables such as "duration of green". Thus, the objectives of this research are itemized as follows:

a. To develop a model to generate a background violation probability, the red-light violation index (RLVI), for urban intersections within DC, with emphasis on variables that could be observed in the field.

b. To determine the effect of vehicles per hour green, lane configuration, intersection width, duration of green and posted speed limit, on the background violation rate. 
A fundamental premise of this research is that all signalized intersections would experience some RLVs, the majority of which are not documented, and that there is a background level of RLVs that could be established for intersections.

\section{Literature review}

Red-light running (RLR) has been a problem for many decades, which dates back to the time when the first modern traffic signals were used in Ohio to control the movement of vehicles and to lower the accident frequency in Cleveland [1]. Since then, departments of transportation (DOTs), and their equivalent agencies around the world, have been implementing diverse methods, including improvements in signal timing, and implementation of RLCs, in order to reduce Red-Light Violations (RLV).

The United States Department of Transportation (USDOT) has approached the problem of red-light-running from two avenues: the Federal Highway Administration (FHWA) addressed the quality of traffic engineering infrastructure and systems for mitigating RLR, and the National Highway Traffic Safety Administration (NHTSA), which looked at the effects of driver inattention, and related human factors on RLR [2].

In terms of countermeasures, the solution to RLR primarily falls into two broad categories: enforcement countermeasures and engineering countermeasures [3]. Enforcement countermeasures involve encouraging drivers to adhere to the traffic laws through use of citations and fines, while engineering countermeasures are geared toward improving driver awareness of traffic signals or reducing the potential of drivers running the red light. Engineering countermeasures hold greater promise than those which involve enforcement, considering the legal and political challenges of automated enforcement [3].

In a 1999 citywide survey conducted in the District of Columbia, residents identified unsafe driving, including running red-lights and stop signs, as their number one public safety concern [4]. Since then RLCs have been implemented throughout the District of Columbia, and evaluation statistics indicated a net decrease of $75 \%$ in violations captured across the respective intersections at which RLCs were installed [5]. However, various studies across the world have challenged the overall effectiveness of RLCs once implemented, given documented costs to the agency, increased collisions of another kind, and challenges by violators who are cited. In light of this, some professionals hold the view that violations may be corrected by other means, based on an assessment of red-light running and crash potential of an intersection. Such assessments, must confirm the existence of a problem of RLVs so that appropriate intervention, if necessary, could be prescribed.

Pietrucha et al. [6] performed a study on motorist compliance with Traffic Control Devices (TCDs), including stop signs and traffic signals [6]. Of the 120 drivers surveyed, 38\% reported having run a red light at some point in their driving careers, and $18 \%$ of these drivers reported doing so at least once a week. For chronic violators, that is those who received a certain number of violation points, $54 \%$ reported running red lights, with $24 \%$ reporting doing so on a 
weekly basis. Forty six percent (46\%) reported personal reasons for running red lights; i.e., "being in a hurry" [6].

An analysis of 156 sites across the US revealed that of the 79,055 vehicles observed, there were 688 violations [7]. The most common maneuver was straight crossing-path (364), followed by left turns (210) and then right turns (114). A 2007 report by the NHTSA suggested that intersection crossing path crashes account for approximately $25 \%$ of all police-reported crashes in the United States each year [7]. They also account for $27 \%$ of all crash-related delays and over $\$ 47$ billion in costs.

It should be noted that crashes caused by running red lights are more deadly and damaging than other types of crashes at signalized intersections [8]. Studies show that the number of RLVs does not necessarily correlate to the number of crashes caused by same [8].

Yan et al. [9] found that the risk of red-light running crashes were related to seven environmental factors - number of lanes, crash time, weather, highway character, day of week, urban/rural, and speed limit; four driver factors - driver age, alcohol/drug use, physical defect; and driver residence, and the type of vehicle [9]. Several of these factors were shown to modulate the effects of other factors, i.e. exhibit an interaction with other factors.

Zimmerman and Bonneson [10] discussed 63 crossing-path crashes at a signalized intersection where red-light violation was a factor. They found that violations occurring more than 5 seconds into the time-into-red tend to be exclusively straight crossing-path crashes, whereas violations occurring fewer than the 5 seconds into time-into-red tend to be exclusively left turn approach (LTAP) crashes [10].

A 2006 NHTSA report indicated that a decrease in red light running was found to be associated with the following factors: [3]

- A decrease in approach flow rate

- An increase in yellow duration

- A decrease in speed

- An increase in clearance path length (i.e. a wider intersection)

- A decrease in platoon density, and

- The addition of signal head back plates.

To combat the problem of violations, when they are suspected to be frequent, the NHTSA report recommended the following actions:

- Confirm the extent of the problem through the computation of the expected frequency of red-light running for the subject location (either by the number of violations per 1,000 vehicles, or number of violators per 100 cycles).

- Compute a "ranking index" that indicates whether the site is truly a problem location [3].

The Red-Light-Running Handbook, developed by Bonnesson and Zimmerman [11] for the Texas Department of Transportation, suggests guidelines for identifying and treating locations that have an unusually large number of red-light violations or related crashes. Separate guidelines are presented for the treatment of red-light problems at individual intersections and 
within entire cities [11]. The method employed the Texas Red-light-running Evaluation and Analysis Tool, (TREAT), to identify problems of red-lightrelated safety based on crash data for an intersection, approach or jurisdiction and comparing it with the average annual crash frequency of similar intersection approaches. If the subject approach had significantly more crashes than similar approaches, then it was identified as having potential benefit from treatment. For a local intersection, it was based on the observed frequency of red-light violations [11]. TREAT was developed using prediction equations calibrated from data for intersections located in urban areas. Therefore the procedure is most applicable to drivers travelling through the intersection (no turns) and urban and suburban intersections. Data for evaluating the RLV problem, according to the TREAT method, includes the following:

1. Traffic Characteristics: Volume, $85^{\text {th }}$ percentile approach speed, heavyvehicle percentage.

2. Traffic Control: Posted speed limit.

3. Signal Operation: Signal cycle length, green phase duration, multi-loop advance detection, approach control mode, left-turn phasing.

4. Motorist information: Signal visibility, signal conspicuity, advance warning signs.

5. Traffic Operation: Approach delay, signal coordination.

6. Geometry: Approach through lanes, approach grade, clearance path length.

7. Red-light Violations: Violation analysis time period, through-vehicle violations during study period.

8. Crash History: Crash analysis time period, crash distribution.

The model developed based on those variables was

$$
\left.\mathrm{E}[\mathrm{R}]=\mathrm{Q} / \mathrm{C}(1 / 1.26) \ln \left[1+\mathrm{e}^{(2.47-1.26 \mathrm{Ye}-0.855 \mathrm{Tct}+0.0545 \mathrm{HV}+0.0693 \mathrm{~V}}{ }_{85}+0.451 \mathrm{fx}-0.414 \mathrm{Bp}\right)\right]
$$

where

$E[R]=$ expected violations for the intersection based on eight variables described above;

$Q$ = approach flow rate, $\mathrm{veh} / \mathrm{h}$;

$C=$ cycle length, $\mathrm{s}$;

$Y e=$ effective yellow duration due to advance detector operation (s),

$T c t=$ clearance time, $\mathrm{s}$;

$H V=$ heavy-vehicle percentage, $\%$;

$V_{85}=85^{\text {th }}$ percentile speed, $\mathrm{mph}$;

$F x=$ overflow delay factor;

$B p=$ presence of back plates on the signal heads, $(1$ if present, 0 if not present).

An index was then developed as follows:

where

$$
\text { Index }=(\mathrm{E}[\mathrm{R} / \mathrm{x}]-\mathrm{E}[\mathrm{R}]) / \sqrt{ }\left(\sigma_{\mathrm{R} / \mathrm{x}}^{2}+\sigma_{\mathrm{R}}^{2}\right)
$$




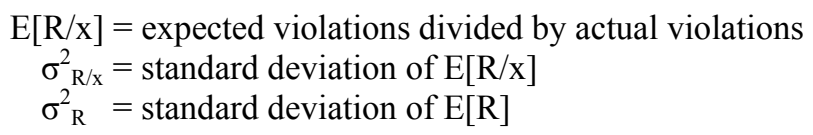

If the index developed was negative, then the intersection would be described as operating at or below the expected RLV frequency. If the index was positive, then the intersection would be operating above the expected RLV frequency, and countermeasures could be recommended based on the degree of positivity. This model has been included in a "Red-Light-Running Handbook" so that engineers may have a framework in which a RLV problem could be identified and treated [11].

As the general literature suggests, there are numerous factors that contribute to RLV. All signals would be violated by at least one driver; this includes unintentional violations. Thus there is always a background level of violation. Apart from a select few studies, the general literature is silent on this matter and whether intersection safety improvement could be measured via observed changes in the expected or background status. This research investigates the background red light violation probability (i.e. the red light violation index) and its use in measuring intersection safety from the perspective of compliance with traffic signals.

\section{Research methodology}

Data was collected at one approach of 18 intersections selected for this study using a video camera, for a 2 hour duration, during the morning off-peak period, between 10:00 am and 12 noon where the potential for red light running is high and violators were not influenced by traffic congestions. The off-peak periods were deliberately selected in order to minimize RLVs that are associated with poor traffic operating conditions. The selection of the approach for video-taping was based on volume and having adequate vantage location for positioning the video cameras. The video playback was used to extract the information for vehicles per hour of green (V), duration of green $(\mathrm{G})$, and lane configuration (L). The posted speed limit (S) on the approaches and intersection width (W) were obtained during site visits. The data extracted from the video files is presented in Table 1 .

\subsection{Selection of variables related to $\mathrm{RLV}$}

From the literature it was determined that a number of variables could have an effect on RLV. These range from human factors such as driver age and ability, to engineering properties, such as intersection geometry and lane characteristics. Only the engineering variables highlighted in the literature that would likely have the most significant impact on red light running, as well as those for which the data collection exercise can be reasonably managed, were selected for developing the background violation index for specific types of intersections. These variables are listed and described in Table 1. 
Table 1: $\quad$ Summary of extracted data.

\begin{tabular}{|c|c|c|c|c|c|}
\hline Intersection & VPHG & Green & $\begin{array}{c}\text { Clearance } \\
\text { distance }\end{array}$ & $\begin{array}{c}\text { Approach } \\
\text { Lane } \\
\text { Configuration }\end{array}$ & $\begin{array}{l}\text { Posted } \\
\text { Speed } \\
\text { Limit }\end{array}$ \\
\hline 1 & 319 & 66 & 92 & 2.5 & 25 \\
\hline 2 & 657 & 59 & 90 & 3.5 & 30 \\
\hline 3 & 586 & 49 & 121 & 4.0 & 25 \\
\hline 4 & 593 & 34 & 130 & 2.5 & 30 \\
\hline 5 & 536 & 52 & 143 & 3.0 & 25 \\
\hline 6 & 341 & 51 & 103 & 3.0 & 25 \\
\hline 7 & 536 & 64 & 90 & 2.5 & 30 \\
\hline 8 & 1014 & 63 & 100 & 4.0 & 25 \\
\hline 9 & 659 & 40 & 113 & 3.0 & 25 \\
\hline 10 & 817 & 61 & 85 & 4.0 & 25 \\
\hline 11 & 693 & 66 & 68 & 5.0 & 30 \\
\hline 12 & 382 & 55 & 102 & 2.5 & 25 \\
\hline 13 & 196 & 26 & 118 & 2.0 & 25 \\
\hline 14 & 308 & 55 & 122 & 1.5 & 25 \\
\hline 15 & 900 & 67 & 68 & 4.5 & 15 \\
\hline 16 & 180 & 24 & 72 & 1.5 & 15 \\
\hline 17 & 158 & 46 & 70 & 1.5 & 25 \\
\hline 18 & 727 & 72 & 66 & 3.5 & 25 \\
\hline
\end{tabular}

\subsubsection{Vehicles per hour green of approach}

Vehicle per hour of green interval of approach is defined as the number of vehicles served by the green interval for the approach to the intersection under study. It includes all the vehicles crossing the stop bar on green, and excludes those vehicles that are considered violators under the definition. This variable was chosen on the theory that number of vehicles that utilize the green influence drivers' attitude regarding running the red light. The literature suggests that this variable directly correlates with RLV at urban intersections.

\subsubsection{Lane configuration}

This variable relates to the number and type of lanes at the intersection approach, as well as the number of turns. Each through lane at an intersection was given a code of 1 , and each turning lane was given a code of 0.5 . The values were summed for the total of each type of lane which was used in the analysis. For example, an intersection with two through lanes and one turning lane, would be assigned a code of 2.5 (one for each through lane and 0.5 for the turning lane, which sums up to 2.5). The literature suggests that the number of approach lanes plays a role in RLV as well as the type (straight or turning lanes). Therefore, a combination of these variables is used as an independent variable in this model. 


\subsubsection{Clearance distance}

The longitudinal distance from the approach intersection's stop bar to the opposing stop bar was used as a variable in the analysis. The literature suggests that the wider the intersection, the less likely a motorist would run a red light, assuming signal timing and all other variables were constant.

\subsubsection{Duration of green}

The duration of green refers to the actual green time allocated to an approach at each intersection. The duration of green affects the queue build up at an intersection which impacts the potential of red light running.

\subsection{Regression model}

To develop the RLVI, each of these independent variables were tested against the dependent variable, red light violation probability (RLVP) to determine the degree of correlation. This was conducted using Microsoft EXCEL and SPSS. After a series of data transformations, the following generalized regression model was deemed to be adequate:

$$
(R L V I)=\beta\left(\mathrm{G}, \mathrm{V}^{-1}, \mathrm{~W}, \mathrm{~W}^{2}, \mathrm{~L}, \log (\mathrm{L}), \mathrm{S}\right)+\varepsilon,
$$

where
$R L V I=$ Red Light Violation Index, same as the background RLVP
$\mathrm{G}=$ Duration of Green
$\mathrm{V}=$ Vehicles per Hour Green
$\mathrm{W}=$ Clearance Distance
$\mathrm{L}=$ Lance Configuration
$\mathrm{S}=$ Posted Speed Limit

The constant $\beta$ is a coefficient of the regression model with an associated error of $\varepsilon\left[\varepsilon \sim N\left(0, \sigma^{2}\right)\right]$. The statistical significance of the regression coefficients were tested at $5 \%$ level of significance. Similarly, the overall statistical significance of the regression model for the intersections was tested using the F-test at 5\% level of significance.

After developing the regression model, an analysis of the predicted values from the model was compared to the observed probabilities for RLR to determine whether there is a statistically significant difference between the two data sets. This was done using the Kolmogorov-Smirnov (KS) test, which provides a D statistic, indicating the difference between the data sets. Since the D statistic was less than the expected D statistic for the sample size, and normality was obtained, then it can be said that there was a good fit between the predicted and observed values for RLV.

The number of vehicles running the amber and the red light were also recorded. Vehicles breaching the amber, and caught in the intersection at the commencement of the red interval were recorded in a separate column labeled "amber/red". The summary results are presented in Table 2. The five variables described were then used to develop a regression model, for the RLVI at $95 \%$ confidence. 
Table 2: $\quad$ Observed violations per cycle.

\begin{tabular}{|c|c|c|c|}
\hline Intersection & $\begin{array}{c}\text { Violation } \\
\text { Opportunities }\end{array}$ & $\begin{array}{c}\text { Number of } \\
\text { Violations }\end{array}$ & $\begin{array}{c}\text { Violation } \\
\text { Probability }\end{array}$ \\
\hline 1 & 90 & 22 & 0.244 \\
\hline 2 & 127 & 38 & 0.299 \\
\hline 3 & 242 & 110 & 0.455 \\
\hline 4 & 170 & 80 & 0.471 \\
\hline 5 & 130 & 46 & 0.354 \\
\hline 6 & 58 & 29 & 0.500 \\
\hline 7 & 142 & 48 & 0.338 \\
\hline 8 & 43 & 7 & 0.163 \\
\hline 9 & 132 & 73 & 0.553 \\
\hline 10 & 76 & 23 & 0.303 \\
\hline 11 & 284 & 110 & 0.387 \\
\hline 12 & 126 & 4 & 0.032 \\
\hline 13 & 66 & 21 & 0.318 \\
\hline 14 & 22 & 12 & 0.545 \\
\hline 15 & 245 & 74 & 0.302 \\
\hline 16 & 57 & 10 & 0.175 \\
\hline 17 & 64 & 17 & 0.266 \\
\hline 18 & 106 & 46 & 0.434 \\
\hline
\end{tabular}

\section{Results}

A statistically significant relationship $(p=0.001216)$ was found between the selected independent variables and the dependent variable, RLVP. The resulting regression model is:

$$
\begin{aligned}
R L V P= & -0.0004807 G-99.91795 V^{1}+0.3354 L-2.9455 \log (L)+0.0133962 \mathrm{~W} \\
& -6 \times 10^{-5} \mathrm{~W}^{2}
\end{aligned}
$$

where

$$
\begin{aligned}
& G=\text { Duration of Green } \\
& V=\text { Vehicles per Hour Green } \\
& \text { W = Intersection Width } \\
& L=\text { Lane Configuration } \\
& \text { RLVP = Red-Light Violation Probability }
\end{aligned}
$$

The percentage of variance $\left(R^{2}\right)$ explained by this model was $81 \%$, suggesting that the majority of the causes of red-light-running in the City could be explained by the independent variables. The summary results for the ANOVA are presented in Table 3 . 
Table 3: $\quad$ Summary results for ANOVA and regression statistics.

\begin{tabular}{|c|c|c|c|c|c|c|}
\hline$A N O V A$ & $d f$ & $S S$ & \multicolumn{2}{|c|}{$M S$} & & Significance $F$ \\
\hline Regression & 6 & 0.626318 & \multicolumn{2}{|c|}{0.104386} & 8.64 & 0.00122 \\
\hline Residual & 12 & 0.144956 & \multicolumn{2}{|c|}{0.01208} & & \\
\hline \multirow[t]{6}{*}{ Total } & 18 & 0.771275 & & & & \\
\hline & & \multicolumn{4}{|c|}{ Regression Statistics } & \\
\hline & & \multicolumn{2}{|c|}{ Multiple R } & \multicolumn{2}{|c|}{0.901142} & \\
\hline & & \multicolumn{2}{|c|}{ R Square } & \multicolumn{2}{|c|}{0.812056} & \\
\hline & & \multicolumn{2}{|c|}{$\begin{array}{l}\text { Adjusted R } \\
\text { Square }\end{array}$} & \multicolumn{2}{|c|}{0.650413} & \\
\hline & & \multicolumn{2}{|c|}{ Standard Error } & \multicolumn{2}{|c|}{0.109908} & \\
\hline
\end{tabular}

The resulting regression model was tested using the Kolmogorov-Smirnov (KS) test in order to determine the statistical difference, D, between the predicted values generated by the model, and the observed values. The results indicate that both sets of data, the predicted and observed values, are normally distributed. Additionally, the D statistic was found to be 0.16667 with $p=0.945$. This indicates that there is there is a close fit between the predicted and observed values for RLVI.

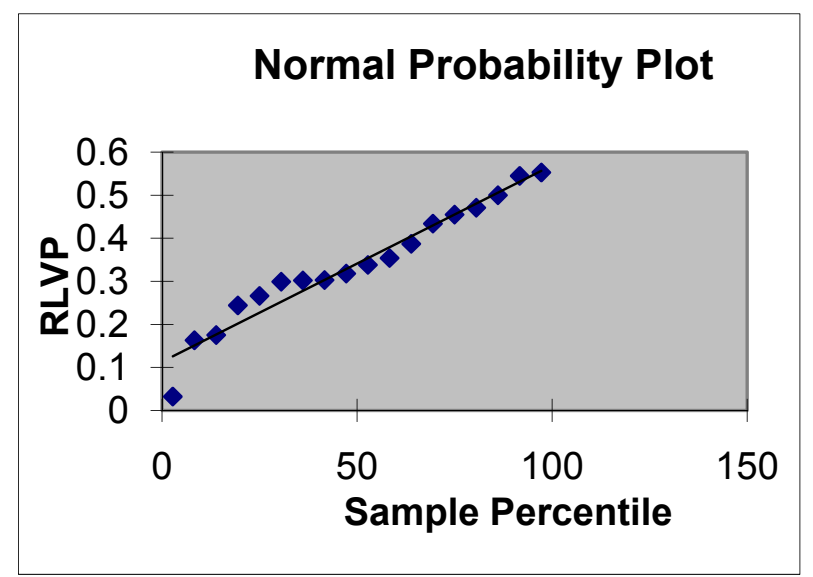

Figure 1: $\quad$ Normal probability plot of RLVP vs sample percentile.

A normal probability plot for the regression model was generated, which produced a straight line graph of approximately 45 degrees, confirming the normality of the distribution. The result is displayed in Figure 1. 


\section{Conclusions}

The analysis show that the intersection-related variables: vehicles per hour green, duration of green, intersection width, lane configuration, and posted speed limit, are strong predictors of red-light running in the District of Columbia. Given that the percentage of variance explained by the model was as high as $81 \%$, it means that modifications to the engineering properties studied in this research may lead to a significant reduction in red-light-running in the District of Columbia.

Although the literature suggests that human factors variables play a significant role in red-light running, the experience in the District of Columbia is that intersection engineering factors could be used to account for the incidence of red-light-running. The potential exists that since the driver-related behavior the variables have a strong correlation with the engineering variables, the model may be accounting for their effect. The RLVI model developed in this research demonstrates that, with only 18 intersections, a reliable model for characterizing the level of RLV for at-grade intersections is achievable. The developed model is applicable to the types of dense urban intersections studied in this research. The model has broad practical appeal since it relies on engineering factors that are easily obtained and available. The research advances the state of knowledge on the question of what benchmark could be used to determine the effectiveness of RLV projects and programs.

Prior to full application of the model, a validation exercise should be undertaken, where data should be collected at a set of test intersections. The predicted values generated could then be compared to observed RLVI to determine the model is validity. As stated in the literature, the causes of red light violations can be vary significantly as one goes from one jurisdiction to the other. Therefore, care should be taken in applying this model in other jurisdictions, and to intersections that are vastly different from these used in this study.

\section{Acknowledgement}

Appreciation is extended to the District Department of Transportation for supporting this study.

\section{References}

[1] USDOT. About Morgan. US Department of Transportation. http://education.dot.gov/aboutmorgan.html .

[2] USDOT. DOT Organizations. US Department of Transportation. http://www.dot.gov/summary.htm .

[3] Bonneson, J., Zimmerman, K., Brewer, M. (2002) "Signalization Countermeasures to Reduce Red-Light-Running," Project Summary Report No. 4027-s, Texas Transportation Institute, The Texas A\&M University System, College Station, TX. 
[4] District of Columbia Metropolitan Police Department. "Automated RedLight Enforcement," http://mpdc.dc.gov/mpdc/cwp/view,a,1240,Q,547886, mpdcNav_GID, 1552,mpdcNav,\%7C, asp.

[5] District of Columbia Metropolitan Police Department. (2007) "Automated Red-Light Enforcement, changes in "Violations Captured" at Camera Locations," $\quad$ http://mpdc.dc.gov/mpdc/frames.asp?doc=/mpdc/lib/mpdc/ info/traffic/pdf/RedLightStats.pdf .

[6] Pietrucha, M., Opiela, T., Knoblauch, K., Crigler, R.L. (1990) "Motorists Compliance with Standard Traffic Control Devices," Vol. 53, No.4, Public Roads.

[7] Suzanne, E. L., et al. (2007) "Intersection Collision Avoidance-Violation Project: Final Project Report," DOT HS 810 749. Virginia Tech Transportation Institute. National Highway Traffic Safety Administration, Washington, DC.

[8] Tijerina, L., Chovan, J., Pierowicz, J., Hendricks, D. (1994) "Examination of Signalized Intersection, Straight Crossing Path Crashes, And Potential IVHS Countermeasures," DOT HS 808 143. National Highway Traffic Safety Administration.

[9] Yan, X., Radwan, E., Birriel, E. (2006) "Left-turn Sight Distance Models for Signalized Intersections with Different Configurations," Section A 10. Advances in Transportation Studies, an International Journal.

[10] Zimmerman, K., Bonneson, J. (2005) "Investigation of the time-into-red for red-light related crashes, " Proceedings of the $84^{\text {th }}$ Annual Meeting of the Transportation Research Board. Transportation Research Board, Washington, DC.

[11] Bonneson, J., Zimmerman, K. (2004) "Red-Light Running Handbook: An Engineer's Guide to Reducing Red-Light-Related Crashes, " FHWA/TX05/0-4196-P1. Texas Transportation Institute, The Texas A\&M University System. Texas Department of Transportation Research and Technology Implementation Office, Austin, TX. 